\title{
Freight-Logistics-as-a-Service - Innovative Geschäftsmodelle für ein datengetriebenes Transportmanagement
}

\author{
Christoph Heinbach (D) S Simon Hagen • Oliver Thomas
}

Eingegangen: 14. Dezember 2020 / Angenommen: 12. März 2021 / Online publiziert: 1. April 2021 (C) Der/die Autor(en) 2021

Zusammenfassung Die Gestaltung digitaler Geschäftsmodelle basiert auf dem disruptiven Potenzial von technologischen Innovationen, das in unterschiedlichen Branchen genutzt wird. Der Logistik kommt in Anbetracht ihrer essentiellen Querschnittfunktion sowie der Entstehung umfangreicher Daten durch den Einsatz verschiedener IT-Systeme für die Abwicklung von Frachttransporten eine besondere Bedeutung zu. Derzeitige Debatten werden mit Blick auf das Cloud-Computing sowie die Merkmale neu entstehender digitaler Plattformen geführt. Insbesondere für das Transportmanagement im Güterkraftverkehr entstehen datenbasierte as-aService Konzepte, die das tradierte Geschäftsmodell von Logistikdienstleistern sowie das Geschäftsfeld der Anbieter von Transportmanagementsystemen (TMS) attackieren. Auffällig ist, dass einer prozessorientierten Betrachtung der cloudbasierten Anwendungssysteme bislang wenig Aufmerksamkeit entgegengebracht wurde. In diesem Beitrag soll ein vertieftes Verständnis für Fracht-Plattformen im Güterverkehr erzeugt werden. Anhand von Experteninterviews wird zunächst das Konzept Freight-Logistics-as-a-Service (FLaaS) für ein datengetriebenes Transportmanagement (DTM) entworfen. Im Anschluss werden auf Grundlage des Business Model Canvas die strategischen Betrachtungen der digitalen Plattformen um Aspekte der Geschäftsmodellentwicklung der Datendienstleister erweitert. Eine intelligente Nutzung des Datenpotentials zeigt sich insbesondere in der Verbesserung der Transpa-

C. Heinbach $(\bowtie) \cdot$ O. Thomas

Fachgebiet Informationsmanagement und Wirtschaftsinformatik, Universität Osnabrück,

Parkstraße 40, 49080 Osnabrück, Deutschland

E-Mail: christoph.heinbach@uni-osnabrueck.de

O. Thomas

E-Mail: oliver.thomas@uni-osnabrueck.de

S. Hagen · O. Thomas

Smart Enterprise Engineering, Deutsches Forschungszentrum für Künstliche Intelligenz (DFKI)

GmbH, Parkstraße 40, 49080 Osnabrück, Deutschland

E-Mail: simon.hagen@dfki.de 
renz sowie der optimierten Auslastung der verfügbaren Transportressourcen. Durch das präsentierte Konzept FLaaS und das betrachtete cloudbasierte Geschäftsmodell werden abschließend strategische Handlungsmaßnahmen formuliert. Die Aspekte Datenkompetenz, Datenstrategie und Kollaboration sind für die Akteure von hoher Relevanz, um eine nachhaltige und wertschöpfende Gestaltung von datengetriebenen Transportketten zu erreichen.

Schlüsselwörter Freight-Logistics-as-a-Service (FLaaS) · Fracht-Plattform • Frachttechnologie $\cdot$ Digitale Transportkette $\cdot$ Datengetriebenes Transportmanagement

\title{
Freight-Logistics-as-a-Service-Innovative Business Models for Data- Driven Transport Management
}

\begin{abstract}
Digital business models are grounded on technological innovations' disruptive potential and applied in various industry sectors. The logistics sector is of particular interest due to its cross-disciplinary role and the generation of data from different IT-systems required to manage freight transportation services. Current discussions regarding cloud computing address the propositions of emerging digital platforms in this area. Hence, data-based as-a-Service concepts arise targeting the transport management for road haulage, which attack traditional business models of logistics service providers and the business field of providers for Transport Management System (TMS). Against this background, a process-oriented approach to cloud-based application systems in the forwarding business did not yet attain close attention to date. This article contributes to a profound understanding of freight platforms in road freight transportation. The Freight-Logistics-as-a-Service (FLaaS) concept for data-driven transportation management (DTM) is outlined based on expert interviews. Digital platforms' strategic dimensions are then extended by business model development aspects related to the data service providers utilizing the Business Model Canvas. Intelligent use of data capabilities is shown in particular by improvements of transparency as well as the optimized utilization of available transport resources. Finally, strategic actions are derived from the presented FLaaS concept and the cloud-based business model. Data competence, data strategy, and collaboration are identified as relevant aspects for the actors to achieve sustainable and value-adding data-driven transport chains.
\end{abstract}

Keywords Freight-Logistics-as-a-Service (FLaaS) · Freight Platform · Freight Technology · Digital Transport Chain · Data-driven Transport Management

\section{Digitale Transformation der Transportlogistik}

Informations- und Kommunikationstechnologien (IKT) durchdringen seit der Entwicklung von ERP-Systemen in den 90er-Jahren und der Einführung von Barcodes zur Verfolgung von Sendungsinformationen die Logistik. Für die Aktivitäten in der Logistik sind Daten, die durch den Einsatz moderner IKT-Systeme die Koordinati- 
on von Material- und Informationsflüssen der Unternehmen unterstützen, essentiell (Niemöller et al. 2017; Hausladen 2020, S. 16). Internet of Things (IoT), CloudComputing und Big Data kommen dabei in der Logistik ebenso zum Einsatz wie analytische und KI-basierte Verfahren, die, basierend auf der Vernetzung physischer Objekte, neue Services für die Beteiligten ermöglichen (Helmke 2019).

Das Transportmanagement gilt in der Logistik als primäre Aufgabe mit hohem Datenpotenzial, deren bestehende Marktchancen von neuen Datendienstleistern durch cloudbasierte Technologien aufgegriffen werden (Dietrich and Fiege 2017; Kille 2018). Im Fokus der digitalen Plattformanbieter steht das Management von Lkw-Transporten. Mit Hilfe der Plattformen werden datenbasierte Services bereitgestellt und die digitale Transformation des Güterverkehrs unterstützt (Göpfert and Seeßle 2019). Als prominentes Leitbild dieser Entwicklung kann die „Uberization“ von Frachtsendungen genannt werden, die das Matching von Angebot und Nachfrage der Transportdienstleistungen mit Hilfe des Konzepts von Multisided-Plattformen (dt. mehrseitige Märkte) realisiert. Gleichwohl steht die Ausrichtung der Services an den Aktivitäten des Transportmanagements im Konflikt zu den traditionellen Anbietern von Transportmanagementsystemen (TMS) (Heinbach et al. 2020). Durch die Verlagerung des Transportmanagements in die Cloud wird den Teilnehmern in der Transportkette die Erzeugung von Mehrwerten ermöglicht. Dieser Aspekt adressiert somit die Value Co-Creation.

Mit dem Einsatz plattformbasierter und betrieblicher Informationssysteme (IS) kann eine durchgängige Digitalisierung der Transportketten erreicht werden (Breusch 2020). Dennoch fällt mit Blick auf die Wissenschaft auf, dass das Potenzial datenbasierter Servicekonzepte in der Transportlogistik bisher primär aus Sicht der Markterscheinungen aufgegriffen wurde (siehe z.B. Elbert and Gleser 2019; Göpfert and Seeßle 2019; Möller et al. 2019). Einer prozessorientierten Betrachtung der cloudbasierten Anwendungssysteme für das operative Transportmanagement wurde bislang wenig Bedeutung beigemessen. Konkret mangelt es an Herangehensweisen und einem objektiven Verständnis der digitalen Plattformlösungen im Kontext eines datengetriebenen Transportmanagements (DTM). Im Rahmen dieses Beitrags wird DTM als Erweiterung des digitalisierten Transportmanagement durch den Einsatz von cloudbasierten Anwendungssystemen definiert. Die Bezeichnung „,datengetrieben“ adressiert folglich die Bereitstellung zusätzlicher digitaler Daten für die Anwender im Rahmen der Transportaktivitäten, um die Entscheidungsqualität für das Transportmanagement durch neue Optionen zu erhöhen. Gleichwohl ist es von großem Interesse, die Merkmale der Geschäftsmodelle für ein DTM zu verstehen, die auf digitalen Plattformen basieren. Die grundlegende Frage, die sich stellt, lautet: Wie kann das cloudbasierte Geschäftsmodell eines Plattformbetreibers gestaltet sein, um ein datengetriebenes Transportmanagement im Güterverkehr zu ermöglichen?

Zur Beantwortung dieser Frage präsentiert dieser Beitrag auf Basis von Experteninterviews das Konzept Freight-Logistics-as-a-Service (FLaaS) und entwirft auf Grundlage des Business Model Canvas ein cloudbasiertes Geschäftsmodell für Plattformbetreiber. Diese erstmalige Konzeption beruht auf den operativen Prozessphasen des Transportmanagements und erweitert die vorrangig strategischen Betrachtungen der digitalen Plattformen in der Transportlogistik um Aspekte der Geschäftsmo- 
dellentwicklung. Es werden weiterhin strategische Handlungsempfehlungen für die Akteure in der Transportkette aufgezeigt, um das Datenpotenzial durch das CloudAngebot für ein DTM zu nutzen.

\section{Intelligentes Frachtgeschäft in der Cloud}

\subsection{Digitale Fracht-Plattformen in der Transportlogistik}

Eine Cloud-Plattform kann als adaptives Softwaresystem verstanden werden, das Kernfunktionen über geeignete Schnittstellen bereitstellt (Tiwana et al. 2010). Digitale Fracht-Plattformen führen mehrere Marktakteure auf informatorischer und kommunikativer Ebene zusammen, sodass eine Wertschöpfung im Transportprozess in Form von Multisided-Märkten durch Interaktionen der Plattformteilnehmer erreicht werden kann (Hagiu und Wright 2015). Ein modernes Paradigma der datengetriebenen Plattformen ist unter dem Begriff Frachttechnologie (engl. FreightTech) bekannt, das eine intelligente Nutzung von Daten als Antrieb zur Entwicklung ökosystemorientierter Plattformkonzepte beschreibt (Roland Berger GmbH 2020). Somit agieren die Plattformbetreiber als Datendienstleister für die Akteure in der Transportkette und greifen den notwendigen Bedarf auf Basis des Serviceleistungs- und Bereitstellungsmodells Software-as-a-Service (SaaS) auf (Göpfert and Seeßle 2019; Möller et al. 2020). Die Plattformen sind zudem in der Lage, bestehende IT-Systeme wie bspw. Telematik zu integrieren und Daten aus unterschiedlichen Quellen zu aggregieren (Bousonville 2017). In der Folge etablieren die neuen Datendienstleister Geschäftsmodelle, die eine Kombination von Datenlieferant und Datenbefähiger darstellen (Wiener et al. 2020).

In Bezug auf das Prozessmodell liegt die Besonderheit dabei in der Interaktionstiefe mit den Akteuren sowie in der Bedeutung der Daten. Die Weitergabe von Transportaufträgen an einen Dritten (Subunternehmer) erweitert auch den Datenaustausch und ermöglicht eine Kollaboration der Dienstleister zur Erfüllung einer Transportaufgabe. Zudem kann der Empfänger in die Kommunikations- und Koordinationsabläufe eingebunden werden, damit Daten genutzt werden, die bspw. eine dynamische Ankunftszeit des Lkw automatisiert berechnen (Abb. 1).

In dieser vereinfachten Darstellung der Prozessschritte, die implizit den Datenaustausch visualisieren, wird eine Transportabwicklung zwischen Verlader und Frachtführer über die Plattform vollständig digital realisiert. Die Integration der Transportprozesse in die Cloud-Architektur ist essentiell, da die operativen Anwendungssysteme für Transportunternehmen durch einen Mangel an Datenstandards sowie einer Vielzahl von IT-Schnittstellen gekennzeichnet sind (Kersten et al. 2017, S. 58). Die Nutzung der digitalen Infrastruktur in der Cloud ist für kleine und mittlere Unternehmen von hoher Signifikanz, um die IT-Fähigkeiten mit den Kunden zu gewährleisten. Zugleich bedrohen die Plattformbetreiber mit ihren datenbasierten und digitalen Service-Angeboten das traditionelle Geschäftsmodell der Logistikdienstleister (Backhaus et al. 2017). 


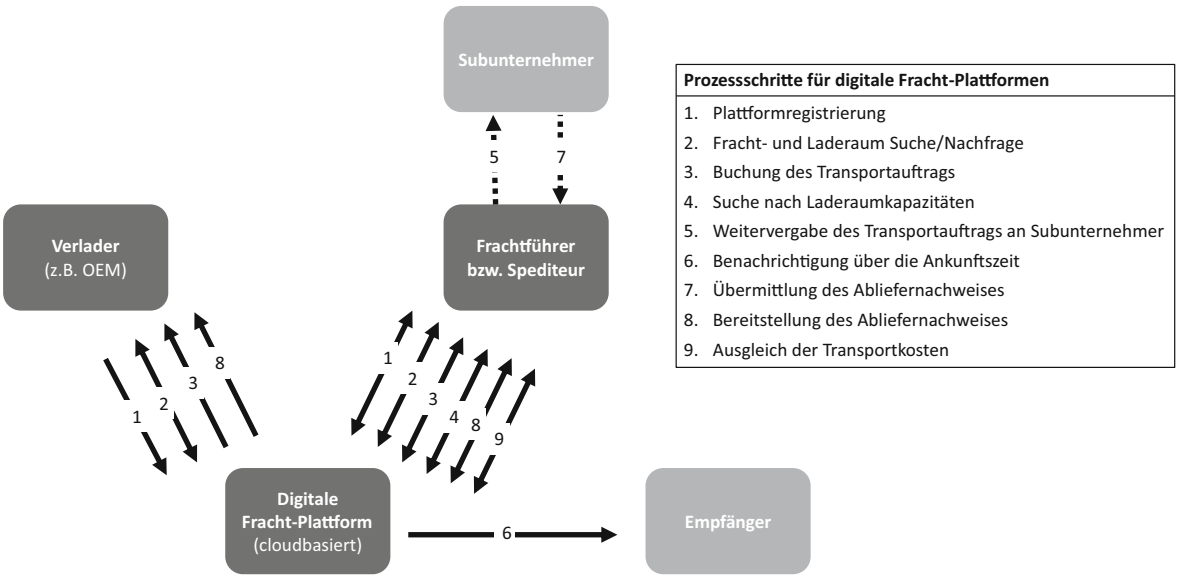

Abb. 1 Prozessmodell digitaler Fracht-Plattformen. (In Anlehnung an Bierwirth et al. 2002)

\subsection{Bestehende Plattformmodelle für ein datengetriebenes Transportmanagement}

Digitale Plattformbetreiber stellen entlang der Transportkette unterschiedliche Service-Angebote bereit, die eine Nutzung der Daten durch die Plattformteilnehmer forcieren. Eine einheitliche und umfassende Darstellung der Datendienstleister für das Transportmanagement im Güterverkehr existiert bislang nicht. Im Folgenden werden daher vier wesentliche Plattformkategorien herausgestellt, die sich mit ihren Modellen im Markt etabliert haben.

1. Frachtenbörsen und digitale Marktplätze

Eine Vermittlung von Angebot und Nachfrage der Fracht- und Transportkapazitäten erfolgt bei diesen Plattformen vorrangig zwischen Verlader und Frachtführer bzw. zwischen den Transportunternehmen. „Matching“-Funktionen ergeben sich hier oftmals über den Preis für einen Transport, wodurch die Plattform den Charakter eines Vergleichsportals zeigt (Dietrich and Fiege 2017).

2. Digitale Transport-Plattformen

Digitale Speditionsdienstleistungen umfassen neben der auftragsbezogenen Buchung von Frachttransporten für den Verlader die vollständige Transportorganisation und -abwicklung einschl. Dokumentation und Abrechnung. Die Anbieter treten als ,digitale Speditionen“ in Erscheinung und sichern sich die notwendigen Transportressourcen über Verträge in direkter Zusammenarbeit mit Frachtführern, um Kostendegressionseffekte gegenüber dem Verlader zu erreichen (Elbert und Gleser 2019).

3. Tender-Plattformen

Ausschreibungen für Frachttransporte für regelmäßige Transporte werden auf eigenen Plattformen mit dem Ziel ermöglicht, den kostengünstigsten und leistungsfähigsten Transportanbieter zu identifizieren. Diese Form der Transportvertragsgestaltung ist in der Transportlogistik unter dem Synonym „Tender“ etabliert. Die 
Anbieter dieser Plattformen ergänzen ihre Services in der Regel um eine weitere Plattform, die eine digitale Abwicklung der Transportaufträge ermöglicht (Wurst 2020).

4. Telematik-Plattformen

Telematik-Systeme leisten im Güterkraftverkehr einen wichtigen Nutzen für ein wirtschaftliches Fahrzeugmanagement. Durch den Einsatz unterschiedlicher Telematik-Systeme verschiedenen Herstellermarken in einem Fuhrpark haben sich Integrationsplattformen entwickelt, die zudem weitere IT-Systeme (z. B. TMS) der Speditionen und Frachtführer integrieren. Durch eine Aggregation der Daten können entsprechende Services für die Kunden bereitgestellt werden (Stölzle et al. 2018, S. 14).

Es wird deutlich, dass das Service-Angebot die Virtualisierung der Unternehmensressourcen unterstützt. Vor dem Hintergrund, dass insbesondere mittelständische Transportanbieter einen Mangel an IT-Kompetenzen aufweisen (Helmke 2019) und zeitgleich eine Vielzahl von IT-Systemen beherrschen müssen (Seifert 2017), bedeuten die Plattformlösungen eine skalierbare und wettbewerbs-relevante Option. Außerdem erhöht die Integration der Transportketten in bestehende Systeme durch Cloud-Architekturen die Flexibilität von Prozessen sowie deren Effizienzgewinne (Kille 2018). Das Angebot der digitalen Services für die Aktivitäten des Transportmanagements führt in der Konsequenz zu einer eigenen Ausprägung assistierender Serviceleistungen durch die Cloud mit positiven Netzwerkeffekten für die Marktakteure (Seiter et al. 2019). Die folgenden Betrachtungen konzentrieren sich auf die Services der digitalen Fracht-Plattformen, die ein DTM ermöglichen.

\section{Freight-Logistics-as-a-Service im Transportmanagement}

In Hinblick auf ein zunehmend cloudbasiertes und IT-gestütztes Transportmanagement von Frachtsendungen wurde eine explorative Untersuchung zur Entwicklung von Cloud-Services in der Logistik durchgeführt. Insgesamt wurden 11 Experten mit unterschiedlichen Positionen (Manager, Abteilungsleiter, Geschäftsführer) aus verschiedenen Organisationen befragt, die als digitale Datendienstleister in der Transportlogistik cloudbasierte Lösungen für das Transportmanagement bereitstellen. Der Schwerpunkt lag bei der Auswahl der Organisation vorrangig auf dem Güterkraftverkehr. Die geführten Gespräche dauerten zwischen 45 min und 113 min. Eine vollständige Übersicht der befragten Experten ist online verfügbar (siehe Onlinematerial 1). Aus den gewonnenen Erkenntnissen leiten die Autoren erstmalig das Konzept Freight-Logistics-as-a-Service (FLaaS) ab. Das Konzept impliziert das digitale Angebot von Cloud-Services zur Unterstützung des Transportmanagements durch Daten im Güterverkehr. Mit Hilfe der Einzelinterviews wurden die bestehenden und zukünftigen Datenservices für das Transportmanagement identifiziert und in das Konzept FLaaS überführt. Die exemplarische Darstellung cloudbasierter Services für ein DTM wurde, ausgehend von der konventionellen Transportdienstleistung, in Abb. 2 den Phasen des Transportmanagement-Prozesses zugeordnet. Die Aktivitäten im Transportmanagement sind angelehnt an die Evaluationsebenen der 


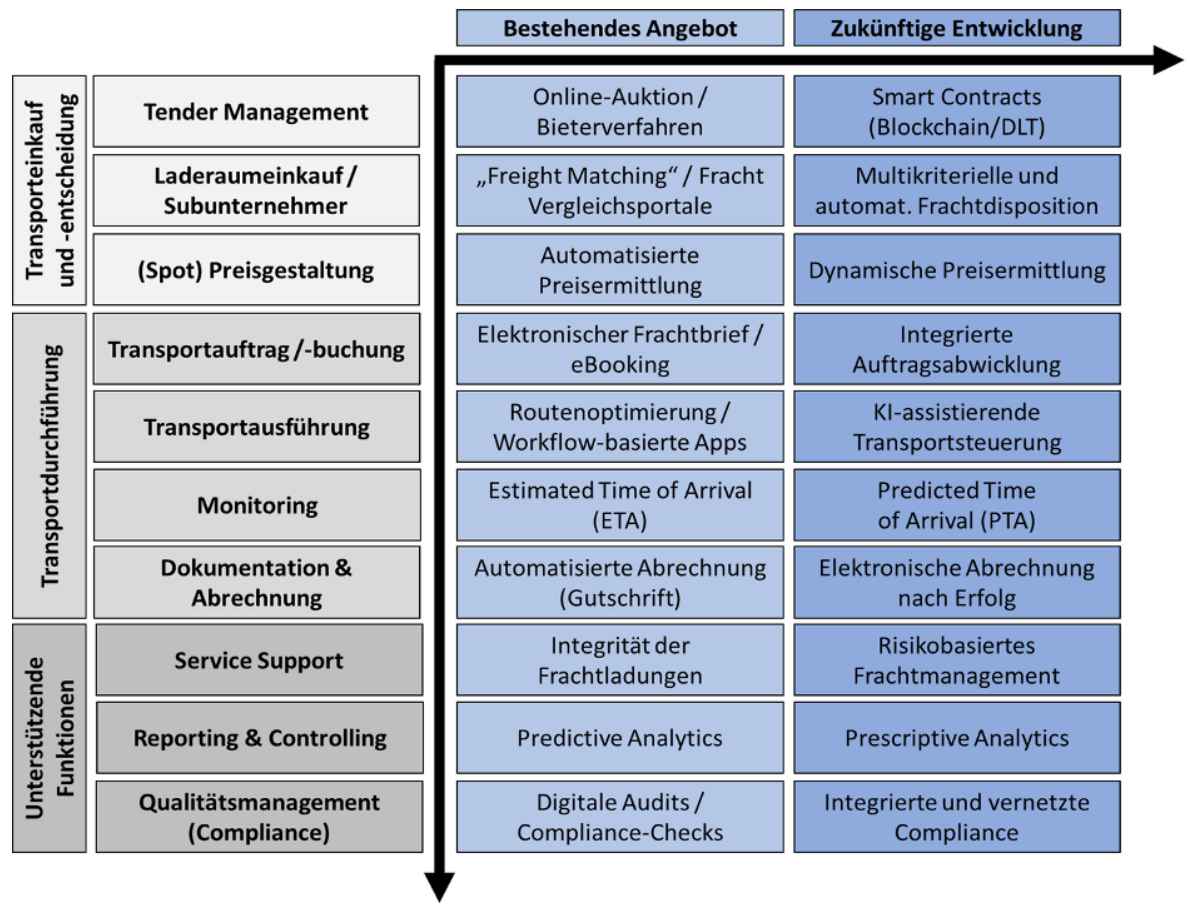

Abb. 2 Freight-Logistics-as-a-Service für das Transportmanagement

TMS-Anwendungen von Gartner (De Muynck et al. 2020) und wurden zudem um die Angaben von Baron et al. (2017) ergänzt.

FLaaS unterstützt ein DTM im Bereich Transporteinkauf und -entscheidung durch ein digitales Vertragsmanagement in Form von Online-Auktionen und Bieterverfahren. Aus den geführten Interviews wurde deutlich, dass ein Laderaumeinkauf sowohl für Auftraggeber als auch für Transportanbieter weiterhin über Vergleichsportale auf Basis von „Freight-Matching“-Prinzipien erfolgt. Eine Aggregation der Daten von Nachfrage- und Anbieterseite gelingt durch den Einsatz intelligenter Algorithmen zur automatisierten Ressourcenallokation. Auch für den freien Transportmarkt - sog. Spot-Geschäfte - ist eine automatisierte Preisermittlung durch den Einsatz von FLaaS möglich. Zukünftig werden Smart-Contracts und der Einsatz von Blockchain-Technologien eine größere Rolle spielen, um den Austausch der Daten abzusichern. Es ist absehbar, dass zukünftig die Transportentscheidung einem multikriteriellen und automatisierten Ansatz folgen wird, der durch äußere Entwicklungen (z. B. CO2-Steuer) beeinflusst wird. Für die (Spot) Preisermittlung wird das dynamische Marktgeschehen durch die verfügbaren Angebote der Frachtladungen und -kapazitäten zukünftig in Echtzeit dargestellt und um weitere Echtzeitparameter (z. B. Diesel- und Strompreis) ergänzt.

Auf Ebene der operativen Transportdurchführung erfolgt die Abwicklung von Transportaufträgen durch FLaaS, wie bspw. die automatisierte Transportbuchung. Ein Austausch von Dokumenten (z. B. Abliefernachweise) wird zudem durch FLaaS 
in Echtzeit ermöglicht, da sich im Rahmen der Prozesskette weitere Automatisierungen (z.B. Rechnungsstellung) ergeben können. Durch den Einsatz mobiler Apps ist eine direkte Verbindung der Transportteilnehmer untereinander möglich, die individuelle Arbeitsabläufe durch „Workflow“-basierte Anwendungen unterstützt. Für das Monitoring der Transportaktivitäten hat sich im Besonderen die voraussichtliche Ankunftszeit (Estimated Time of Arrival - ETA) des Fahrzeugs als Servicedisziplin entwickelt. Mit Blick auf die zukünftigen Entwicklungen ist zu erwarten, dass neben den Integrationsinitiativen von weiteren Servicebereichen (z. B. Palettenmanagement) Methoden der Künstlichen Intelligenz eine größere Bedeutung erlangen werden. Das betrifft sowohl die Unterstützung von Transportentscheidungen bei der Transportausführung als auch die Prognose- und Vorhersagbarkeit von Ereignissen (z.B. Predicted Time of Arrival - PTA). Die Dokumentations- und Abrechnungsmodelle können zukünftig erfolgsbasierte Ansätze beinhalten und das konforme „Verhalten“ eines Marktakteurs (z.B. Lieferqualität, Schadensquote) berücksichtigen.

Die Cloud-Infrastruktur wird von den Plattformbetreibern zudem für die Bereitstellung unterstützender Funktionen für das Transportmanagement genutzt. FLaaS unterstützt eine durchgängige Transparenz von Frachtladungen und damit die Lieferkettenintegrität bei relevanten Ereignissen (z. B. Ankunftszeiten der Sendungen, Güterschäden). Prädiktive Analysen werden eingesetzt, um dem Kunden im Vorfeld Angaben zu liefern, die auf eine Optimierung des eingesetzten Equipments (z. B. Predictive Maintenance beim Lkw) oder auf mögliche Leistungsprobleme (z. B. Einhaltung von Zeitvorgaben) hinweisen. Einen weiteren Einsatz von FLaaS stellen Selfservice-Dienste durch digitale Audits bzw. die Sicherstellung der eigenen Compliance gegenüber Dritten dar. Ein risikobasiertes Frachtmanagement wird sich nach Aussage der Experten zukünftig etablieren. Potenzielle Risiken in der Transportkette können durch FLaaS und die Integration verschiedener Datensysteme zukünftig bewertet werden, um ggf. alternative Verkehrsträger zu erwägen. Zudem wird sich die Vorhersagbarkeit von Ereignissen mit Unterstützung neuer Technologien zu prognosebasierten Modellen erweitern, um die Planungsqualität zwischen den Beteiligten $\mathrm{zu}$ verbessern. FLaaS wird in Zukunft eine integrierte und vernetzte Compliance forcieren, um Transportentscheidungen unter Berücksichtigung weiterer Parameter wie bspw. die Einhaltung von Rechtsvorschriften oder CO2-Emissionen zu treffen.

Das präsentierte Konzept FLaaS zeigt ein enormes Potenzial für ein DTM und die systematische Durchgängigkeit der Prozessorientierung durch Daten impliziert das Vorhandensein eines cloudbasierten Geschäftsmodells, das im Folgenden für die Plattformbetreiber konzipiert wird.

\section{Konzeption des Geschäftsmodells Freight-Logistics-as-a-Service}

Zur Gestaltung innovativer Geschäftsmodelle hat sich die Business Model Canvas (BMC) sowohl in der Praxis als auch in der Wissenschaft durchgesetzt. Auf Basis einer intuitiven Vorgehensweise wird die Betrachtung neun verschiedener Aspekte eines Geschäftsmodells unterstützt (Osterwalder 2004; Osterwalder and Pigneur 


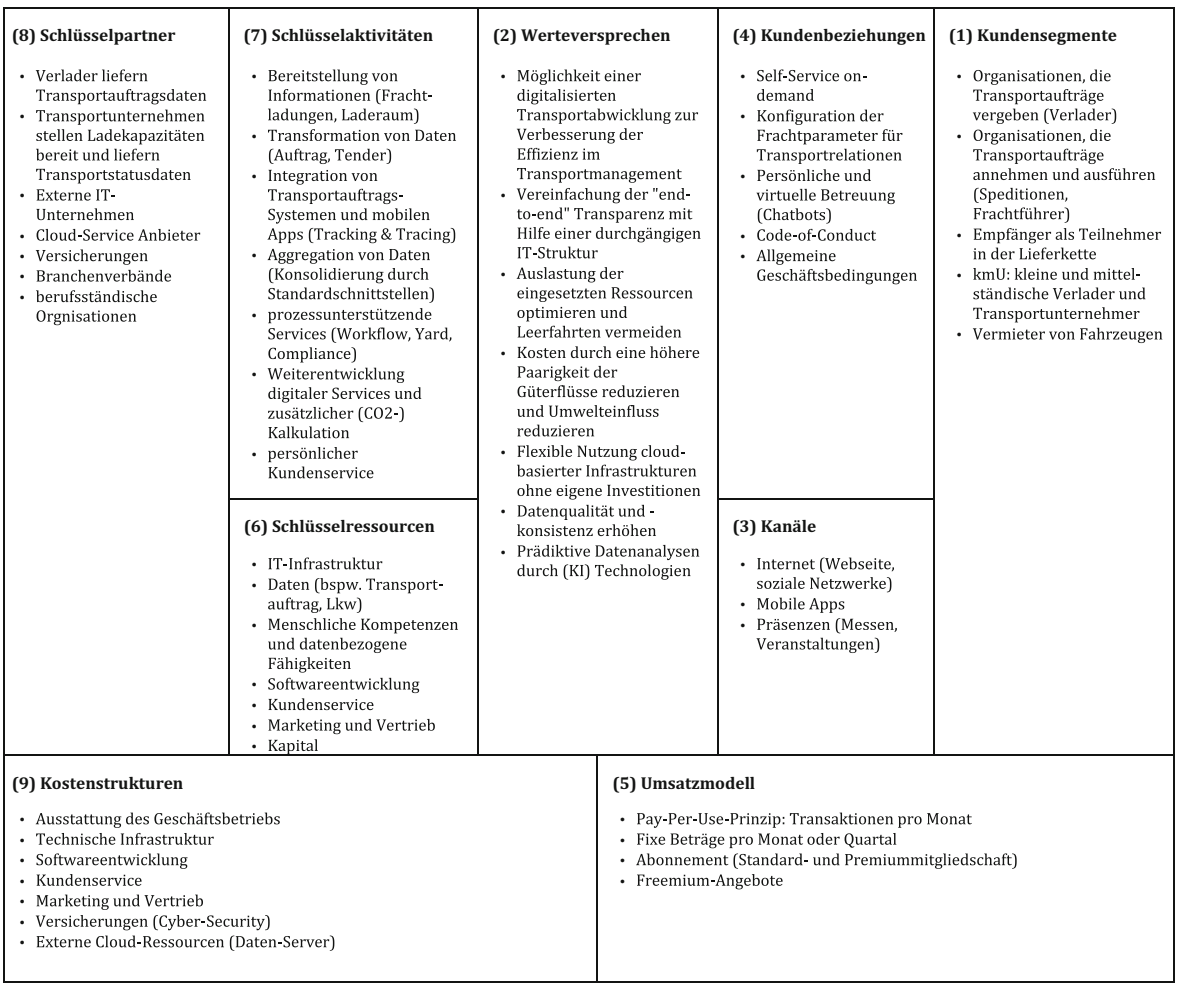

Abb. 3 Freight-Logistics-as-a-Service dargestellt mit Hilfe des Business Model Canvas

2010). Nachfolgend werden diese Aspekte für FLaaS beschrieben und in Abb. 3 in der bekannten Darstellungsweise visualisiert.

\section{Kundensegmente}

Kunden, die FLaaS nutzen, sind Organisationen, die Transportaufträge vergeben und als sog. ,Verlader“ im Transportrecht auftreten (z. B. Händler oder Hersteller bzw. OEM). Des Weiteren werden die Services von Transportunternehmen, Speditionsbetrieben und Transportdienstleistern im Sinne eines „Frachtführers“ genutzt, um die Transportaufträge der Verlader auszuführen. Weitere Kundengruppen entlang der operativen Transportketten sind die Empfänger der Frachtsendungen und die Vermieter von Frachtfahrzeugen.

\section{Werteversprechen}

Die Mehrwerte für die Kunden sind in der Cloud-Infrastruktur zu sehen, die eine weitestgehend datengetriebene Transportabwicklung unterstützt und dadurch Effizienzverbesserungen in den Prozessen erreicht. Durch eine durchgängige ITStruktur in der Transportkette wird die Abwicklung von Geschäftsprozessen transparent und für die Kunden vereinfacht, was sich als Zeitersparnis und höhere Transportqualität für die Kunden darstellt. Mit der Konsolidierung von Angebot und Nachfrage der Transportdienstleistungen werden sowohl die Auslastung 
der eingesetzten Ressourcen optimiert als auch Leer-Kilometer der Fahrzeuge reduziert. Ein zusätzliches Versprechen stellen prädiktive Datenanalysen durch den Einsatz von (KI) Technologien dar (z. B. Lkw-Wartungen, Ankunftszeit beim Empfänger).

3. Kanäle

Anbieter von FLaaS nutzen das Internet und setzen außerdem mobile Apps ein, um mit den Kunden in Kontakt treten zu können. Die Bewerbung für das Wertangebot erfolgt dazu sowohl über die Webseite des Unternehmens als auch über soziale Netzwerke, die den Kunden zu aktuellen Geschehnissen sowie zu den Produkt- und Serviceleistungen informieren. Die Kontaktaufnahmen mit potenziellen Neukunden finden zudem auf Messen und branchenspezifischen Veranstaltungen statt.

4. Kundenbeziehungen

Die Kunden treffen zu Beginn der Beziehung im Self-Service eine Auswahl des Umfangs und der Bestandteile des Wertangebotes „on-demand“. Zudem ist eine Individualisierung durch die Konfiguration der Frachtparameter möglich, die bspw. die Festlegung des Lade- und Lieferorts (sog. Transportrelation), des Ladungstyps sowie der Transportfrequenz umfasst. Eine persönliche oder digital assistierende Unterstützung ist jederzeit für die Kunden verfügbar. Die Annahme des Wertangebots erfolgt mit der Zustimmung der Bedingungen des Anbieters, bspw. durch die Bestätigung eines „Code of Conduct“. Eine Vereinbarung von ServiceLevel-Agreements (SLAs) erfolgt in einzelnen Fällen.

5. Umsatzmodell

Die Leistungen der FLaaS-Anbieter können transaktionsbasiert nach dem PayPer-Use-Prinzip (z. B. monatlich) in Anspruch genommen werden. Ein weiteres Abrechnungsmodell ist die Festlegung fixer Beträge, die in definierten Perioden (z.B. monatlich, quartalsweise) und in Bezug auf die Anzahl von Nutzern entrichtet werden. Es existieren zudem Modelle, die eine Standard- und Premiummitgliedschaft in Abhängigkeit vom genutzten Leistungsumfang unterscheiden und als Abonnement definiert sind. Zusätzliche Differenzierungen umfassen Freemium Angebote, die eine kostenfreie Nutzung von Basisleistungen für die Kunden festlegen und individualisierte Angebote mit kundenspezifischen Konditionen (z. B. Reklamationen, Service-Level).

6. Schlüsselressourcen

Für den Betrieb von FLaaS ist eine IT-Infrastruktur notwendig, die in Rechenzentren bzw. Data-Center die notwendige Hard- und Software betreibt. Daten werden aus unterschiedlichen Bereichen (bspw. Transportauftrag, Lkw) genutzt, um Schlüsselaktivitäten zu ermöglichen. Menschliche Kompetenzen und datenbezogene Fähigkeiten werden in den Bereichen der Softwareentwicklung, im Kundenservice sowie Marketing und Vertrieb benötigt. Die Entwicklung ,intelligenter“ Services mit einem hohen Technologieanteil, die schnell verfügbar sind, erfordert außerdem Kapital, das durch FinTech (Finanzdienstleistungen) bereitgestellt wird.

7. Schlüsselaktivitäten

Für FLaaS werden die folgenden sieben Aktivitäten unterschieden: Eine Bereitstellung von Informationen umfasst das Angebot von Frachtladungen und verfügbarem Laderaum sowie Details zu Transportaufträgen und Compliance. Die 
Transformation von Daten erfolgt durch die digitale Verarbeitung von Transportauftragsinformationen durch digitale Speditionsleistungen. Eine Integration von IT-Systemen (z. B. TMS) ermöglicht eine durchgängige Verfügbarkeit von Informationen zum Status der Lieferung. Eine Aggregation von Daten umfasst die Konsolidierung von Daten verschiedener IT-Systeme (z. B. Telematik, ERP). Ein DTM wird durch prozessunterstützende Services ergänzt, die bspw. den Workflow oder das Yard Management betreffen. Das Wertangebot für die Kunden wird durch die Weiterentwicklung digitaler Services und zusätzlicher Dienste erweitert (z.B. CO2-Kalkulation pro Transport, Inkasso-Service). Ein persönlicher Kundenservice unterstützt die Kunden bei der Implementierung und Nutzung der FLaaSLösungen sowie bei der Analyse von Potenzialen durch Daten.

8. Schlüsselpartner

Daten und Informationen sind von den Kunden sowohl in Bezug auf die Transportaufträge (Verlader) als auch zum Laderaum (Frachtführer) unerlässlich, um das Wertangebot von FLaaS zu realisieren. Insofern kann FLaaS als Sonderform der Co-Creation betrachtet werden. Durch die Zusammenarbeit mit weiteren ITUnternehmen wird mit Hilfe der Integration weiterer Technologien die echtzeitbasierte Frachtverfolgung und eine verbesserte Prognose zur Ankunftszeit der Transporte erreicht. Zusätzliche Schlüsselpartner sind weitere Anbieter von Cloud-Services, von denen zusätzliche Speicherressourcen für die Softwareentwicklung genutzt werden können. Mit der Kooperation von Versicherungen, Branchenverbänden und berufsständischen Organisationen ergeben sich weitere Schlüsselpartner.

9. Kostenstrukturen

Die Kostenstruktur beinhaltet die Ausstattung des Geschäftsbetriebs sowie die Anschaffung der technischen Infrastruktur. Kosten entstehen den Datendienstleistern weiterhin durch den Einsatz von Personal in den Unternehmensbereichen, insbesondere in der Softwareentwicklung. Außerdem umfassen Kostenelemente Lizenzen (z.B. Software) und Versicherungen (z.B. Cyber-Security). Variable Kosten entstehen den Anbietern durch die flexible Nutzung externer Cloud-Ressourcen (z.B. Daten-Server), die sich ebenfalls an dem Pay-Per-Use-Prinzip orientieren.

\section{Strategische Handlungsempfehlungen}

Auf Basis empirischer Untersuchungen konnte das cloudbasierte Geschäftsmodell Freight-Logistics-as-a-Service (FLaaS) entworfen werden. Das präsentierte Modell schließt die Integration eines spezialisierten Datendienstleisters als Bindeglied zwischen Transportauftraggebern und -nehmern sowie weiteren Akteuren in der Transportkette ein. Die eingangs formulierte Frage konnte durch das konzipierte Business Model Canvas beantwortet werden. Um mit dem vorgestellten Cloud-Konzept FLaaS und dem betrachteten cloudbasierten Geschäftsmodell ein datengetriebenes Transportmanagement (DTM) strategisch zu gestalten und zu unterstützen, ergeben sich für die Akteure unterschiedliche Handlungsoptionen: 
1. Identifikation der IT- und Datenkompetenzen

Die Handhabbarkeit von verfügbaren Daten wird in Zukunft für ein DTM von erfolgskritischer Bedeutung sein. Akteure in der Transportlogistik sollten daher ihre IT-Standards und Schnittstellen überprüfen, um einen durchgängigen Datenaustausch durch den Einsatz von Cloud-Lösungen ,end-to-end“ zu ermöglichen. Dazu müssen Verlader und Transportunternehmen die Interoperabilität ihrer Systeme gemeinsam auf den Prüfstand stellen. Der Einsatz von gängigen TMS und ERP-Systemen bietet einen Ausgangspunkt, um die Integration weiterer Systeme (z.B. Telematik, WMS) zu bewerten. Analoge Informationen, die darüber hinaus noch in „Silos“ existieren, müssen zudem digitalisiert werden. Die dafür notwendigen Kompetenzen müssen systematisch auf allen Unternehmensebenen mit Unterstützung der Fach- und Führungskräfte entwickelt werden. Der „Data Scientist“ im Unternehmen wird zukünftig die Integration der Transportketten in bestehende Systeme durch Cloud-Architekturen maßgeblich mitgestalten. Unternehmen sind durch den Aufbau eigener Cloud-Kompetenzen folglich in der Lage, eine ,make or join"-Entscheidung zu treffen, die eine Bewertung für den Aufbau einer eigenen digitalen Plattform oder für die Nutzung eines bestehenden Cloud-Angebots durch existierende Plattformen umfasst.

2. Entwicklung einer cloudbasierten Datenstrategie

Daten müssen durch das Management in den Unternehmen als Schlüsselressource in Transportketten in Form einer cloudbasierten Datenstrategie formuliert werden, damit ein regulierter und digitaler Unternehmenswandel ermöglicht wird. Mögliche Aspekte betreffen die Korrespondenz mit der Unternehmensstrategie, die Governance, die Datenhoheit- und -sicherheit, das Risikomanagement sowie die Datenkultur. Im Umgang mit Cloud-Lösungen sollten Verlader strategische Schlüsselkriterien bei der Dienstleisterauswahl berücksichtigen, die eine bisher eher preisgetriebene Entscheidungssicht im Frachteinkauf erweitert. Als Beispiel könnte ein cloudbasiertes Compliance-System mit einer Tender-Plattform kombiniert werden. Transportunternehmen müssen, basierend auf den festgestellten ITLücken, einen „,make or join“-Ansatz definieren und entscheiden, welche CloudServices die Leistungsfähigkeit und Wettbewerbsposition nachhaltig unterstützen. Dabei sind Lock-in Effekte und die parallele Teilnahme an verschiedenen Plattformen (,Multihoming“) zu berücksichtigen.

3. Fokussierung eines kollaborativen und datengetriebenen Transportmanagements Auf dem Weg zu einer datengetriebenen Transportlogistik agieren die Transportunternehmen in einer zunehmend plattformökonomisch dominierten Umwelt mit unterschiedlichen Chancen und Risiken. Eine Konsolidierung von Frachtressourcen verschiedener Transportunternehmen kann die Wirtschaftlichkeit und Wettbewerbsfähigkeit erhöhen. Der Vision von Heinbach et al. (2020) folgend könnte mit Hilfe der Cloud-Technologien durch die Aggregation der Ressourcen ein kollaboratives DTM erreicht werden. Dieser Schritt erfordert ein Umdenken von festen und bestehenden Leistungsstrukturen hin zu adaptiven und agilen Service-Systemen. Kollaborationsmodelle mit Verladern, Empfängern und weiteren Schlüsselpartnern sind vor dem Hintergrund wandlungsfähiger und flexibler Logistikkonzepte zu bewerten, die damit einen Freiraum für prototypische Entwicklungen 
schaffen. Ergänzende intelligente und datenbasierte Dienstleistungen können in Form von as-a-Service-Konzepten in der Cloud entstehen. Prädiktive Datenanalysen und der Einsatz von KI werden zukünftige Ideengeber sein, um das Innovationspotenzial konsequent zu nutzen.

\section{Fazit}

Das in diesem Beitrag betrachtete Servicekonzept Freight-Logistics-as-a-Service (FLaaS) bietet für das datengetriebene Transportmanagement (DTM) im Güterkraftverkehr bemerkenswerte Potenziale und kann als neuartige Geschäftslogik verstanden werden. Durch die Konzeption des cloudbasierten Geschäftsmodells wurden die bislang primär als Frachttechnologie beschriebenen Ausprägungen der digitalen Transformation in der Transportlogistik um die Aspekte der Geschäftsmodellentwicklung erweitert. Anhand des Business Model Canvas konnte, basierend auf Experteninterviews, gezeigt werden, wie die Eigenschaften des Cloud-Computing für das DTM genutzt werden können. Dieser erste, aber erforderliche Entwurf fungiert als Grundlage für die Entwicklung weiterer Geschäftsmodelle, die im Kontext intermodaler Transportketten mit unterschiedlichen Wertschöpfungsstufen entstehen können.

Die Cloud-Technologie ist in der Lage, eine Synchronisierung der Güter- und Informationsflüsse zwischen Transportanbietern und -nachfragern herbeizuführen und durch die Integration der Systeme durchgängige Prozesse darzustellen. Allerdings stellt die neue Form der Transparenz die traditionellen Geschäftsmodelle der Transportdienstleister vor neue Herausforderungen, da die schnelle und unkomplizierte Bereitstellung datengetriebener Transportservices neue Entscheidungsqualitäten seitens der Verlader erzeugen. Die komfortable Situation für die Auftraggeber zeigt sich aktuell darin, dass Transportverträge bedeutend kürzeren Laufzeiten unterliegen bzw. ad hoc mit den Transportdienstleistern gekündigt werden (Wurst 2020).

Die große Herausforderung wird für die Akteure in der Transportkette darin bestehen, die Möglichkeiten einer datengetriebenen und vernetzten Transportlogistik durch den Einsatz neuer Technologien zu erfassen und strategisch im Unternehmen auf allen Ebenen nachhaltig zu verankern. Der Fokus für weitere Untersuchungen muss sich nach Auffassung der Autoren stärker mit den cloudbasierten Potenzialen für ein DTM aus Sicht der Transportdienstleister sowie der „Digital Leadership“ im Unternehmen beschäftigen, um eigene digitale Strategien und Konzepte zu entwickeln, bevor bestehende Geschäftsmodelle drohen, vollständig obsolet zu werden.

Zusatzmaterial online Zusätzliche Informationen sind in der Online-Version dieses Artikels (https://doi. org/10.1365/s40702-021-00718-y) enthalten.

Funding Open Access funding enabled and organized by Projekt DEAL.

Open Access Dieser Artikel wird unter der Creative Commons Namensnennung 4.0 International Lizenz veröffentlicht, welche die Nutzung, Vervielfältigung, Bearbeitung, Verbreitung und Wiedergabe in jeglichem Medium und Format erlaubt, sofern Sie den/die ursprünglichen Autor(en) und die Quelle ordnungsgemäß nennen, einen Link zur Creative Commons Lizenz beifügen und angeben, ob Änderungen vorgenommen wurden. 
Die in diesem Artikel enthaltenen Bilder und sonstiges Drittmaterial unterliegen ebenfalls der genannten Creative Commons Lizenz, sofern sich aus der Abbildungslegende nichts anderes ergibt. Sofern das betreffende Material nicht unter der genannten Creative Commons Lizenz steht und die betreffende Handlung nicht nach gesetzlichen Vorschriften erlaubt ist, ist für die oben aufgeführten Weiterverwendungen des Materials die Einwilligung des jeweiligen Rechteinhabers einzuholen.

Weitere Details zur Lizenz entnehmen Sie bitte der Lizenzinformation auf http://creativecommons.org/ licenses/by/4.0/deed.de.

\section{Literatur}

Backhaus A, Krol F, Meisner M, Stölzle W (2017) Digitalisierung in der Logistik: vom Hype zur Marktrelevanz? In: Logistik im Spannungsfeld der Politik. DVV Media Group, Hamburg, S 40-61

Baron R, Zintel M, Zieris M, Mikulla D (2017) Digital platforms in freight transportation-A true industry disruptor? Arthur D. Little

Bierwirth C, Schneider S, Kopfer H (2002) Elektronische Transportmärkte Aufgaben, Entwicklungsstand und Gestaltungsoptionen. Wirtschaftsinf 44:335-344. https://doi.org/10.1007/BF03250858

Bousonville T (2017) Anwendungsfälle im Transport. In: Logistik 4.0. Springer, Wiesbaden, S 27-34

Breusch M (2020) Supply Chain 4.0 - Voraussetzungen für die Digitalisierung in der Lager- und Transportlogistik. In: Logistik - die unterschätzte Zukunftsindustrie. Springer, Berlin Heidelberg, S 71-82

Dietrich A, Fiege F (2017) Digitale Transformation des Speditionsgeschäfts umfasst mehr als Spedition 4.0. Wirtschaftsinformatik Manag 9:36-45. https://doi.org/10.1007/-s35764-017-0058-6

Elbert R, Gleser M (2019) Digital forwarders: a market oriented taxonomy. In: Bierwirth C, Kirschstein T, Sackmann D (Hrsg) Logistics Management. Springer, Cham, S 19-31

Göpfert I, Seeßle P (2019) Innovative Startups in der Logistikbranche - Eine Betrachtung der neuen Marktteilnehmer und empirische Erkenntnisse einer Fragebogenstudie. In: Logistik der Zukunft-Logistics for the Future. Springer, Berlin Heidelberg, S 253-280

Hagiu A, Wright J (2015) Multi-sided platforms. Int J Ind Organ 43:162-174

Hausladen I (2020) IT-gestützte Logistik: Systeme - Prozesse - Anwendungen. Springer, Wiesbaden

Heinbach C, Kammler F, Thomas O (2020) Smart Forwarding - Datengetriebene Wertschöpfung in der Logistikkette. Wirtsch Inform Manag. https://doi.org/10.1365/s35764-020-00294-8

Helmke B (2019) Digitalisierung in der Logistik. In: Hartel DH (Hrsg) Projektmanagement in Logistik und Supply Chain Management. Springer, Wiesbaden, S 183-207

Kersten W, Seiter M, von See B et al (2017) Chancen der digitalen Transformation: Trends und Strategien in Logistik und Supply Chain Management. DVV Media Group GmbH, Hamburg

Kille C (2018) Digital Logistics. In: Fend L, Hofmann J (Hrsg) Digitalisierung in Industrie-, Handels- und Dienstleistungsunternehmen. Springer, Wiesbaden, S 125-137

Möller F, Bauhaus H, Hoffmann C et al (2019) Archetypes of Digital Business Models in Logistics StartUps. In: Twenty-Seventh European Conference on Information Systems (ECIS2019). StockholmUppsala, Sweden

Möller F, Stachon M, Hoffmann C et al (2020) Data-driven business models in logistics: a taxonomy of optimization and visibility services. In: Proceedings of the 53rd Hawaii international conference on system sciences Hawaii. In, , S 5379-5388

De Muynck B, Johns B, Sanchez Duran O (2020) Magic quadrant for transportation management systems. Gartner, Stamford

Niemöller C, Zobel B, Berkemeier L et al (2017) Sind Smart Glasses die Zukunft der Digitalisierung von Arbeitsprozessen? Explorative Fallstudien zukünftiger Einsatzszenarien in der Logistik. In: Wirtschaftsinformatik (WI2017) St. Gallen, S 410-424

Osterwalder A (2004) The business model ontology a proposition in a design science approach. PhD Thesis, Université de Lausanne, Faculté des hautes études commerciales

Osterwalder A, Pigneur Y (2010) Business model generation: a handbook for visionaries, game changers, and challengers. Wiley, Hoboken

Roland Berger GmbH (2020) FreightTech - Treiber für die Logistik der Zukunft. Whitepaper, München

Seifert H (2017) Die Digitalisierungskompetenz als Kernkompetenz des Logistikdienstleisters. In: Logistik im Spannungsfeld der Politik. DVV Media Group, Hamburg, S 111-115 
Seiter M, Autenrieth P, Schüler F (2019) Logistikdienstleister im Zeitalter digitaler Plattformen. In: Schröder M, Wegner K (Hrsg) Logistik im Wandel der Zeit - Von der Produktionssteuerung zu vernetzten Supply Chains. Springer, Wiesbaden, S 585-600

Stölzle W, Schmidt T, Kille C et al (2018) Digitalisierungswerkzeuge in der Logistik: Einsatzpotenziale, Reifegrad und Wertbeitrag: Impulse für Investitionsentscheidungen in die Digitalisierung - Erfolgsgeschichten und aktuelle Herausforderungen. Cuvillier Verlag, St. Gallen

Tiwana A, Konsynski B, Bush AA (2010) Platform evolution: coevolution of platform architecture, governance, and environmental dynamics. Inf Syst Res 21:675-687. https://doi.org/10.1287/isre.1100. 0323

Wiener M, Saunders C, Marabelli M (2020) Big-data business models: a critical literature review and multiperspective research framework. J Inf Technol 35:66-91

Wurst C (2020) Chancen von Logistik 4.0 nutzen. Control Manag Rev 64:34-39. https://doi.org/10.1007/ s12176-019-0084-8 